\title{
Treatment Pattern for Advanced Gastric Cancer in Japan and Factors Associated with Sequential Treatment: A Retrospective Administrative Claims Database Study
}

\author{
Yoshito Komatsu (D) - Shuichi Hironaka • Yoshinori Tanizawa • \\ Zhihong Cai · Yongzhe Piao • Narikazu Boku
}

Received: July 28, 2021 / Accepted: September 22, 2021 / Published online: October 29, 2021

(C) The Author(s) 2021

\begin{abstract}
Introduction: Clinical trials have proven the efficacy and safety of new therapies for advanced gastric cancer (AGC), but how those therapies are used in the real world is poorly
\end{abstract}

Supplementary Information The online version contains supplementary material available at https:// doi.org/10.1007/s12325-021-01931-3.

Y. Komatsu $(\bowtie)$

Department of Cancer Chemotherapy, Hokkaido

University Hospital Cancer Center, Kita 14, Nishi 5,

Kita-ku, Sapporo, Hokkaido 060-8648, Japan

e-mail: ykomatsu@ac.cyberhome.ne.jp

S. Hironaka

Department of Medical Oncology and Hematology,

Oita University, Faculty of Medicine, Oita, Japan

e-mail: shironaka@oita-u.ac.jp

Y. Tanizawa $\cdot$ Z. Cai $\cdot$ Y. Piao

Medicines Development Unit-Japan, Eli Lilly Japan

K.K., Kobe, Japan

Y. Tanizawa

e-mail: tanizawa_yoshinori@lilly.com

Z. Cai

e-mail: cai_zhihong@lilly.com

Y. Piao

e-mail: piao_yongzhe@lilly.com

N. Boku

IMS Hospital, Institute of Medical Science, The

University of Tokyo, Tokyo, Japan

e-mail: nboku@ims.u-tokyo.ac described. Real-world treatment patterns of antitumor therapies and factors associated with overall therapy duration in patients with AGC in Japan were investigated.

Methods: This retrospective cohort study used a Japanese administrative claims database (June 2014 to September 2019). Patients with AGC who started the guideline-recommended firstline combination regimens with platinum and fluoropyrimidine agents between June 2015 and July 2019 were included. Cox regression analysis was performed to identify factors associated with overall therapy duration (first line to last administration of guideline-listed agent).

Results: Of the 10,581 patients included, the most common first-line combination regimen without trastuzumab was S-1 plus oxaliplatin (4327/9069 patients; $47.7 \%$ ) and with trastuzumab was capecitabine plus cisplatin (608/1512 patients; 40.2\%). Most common second- and third-line regimens were ramucirumab plus taxane (3650/5358 patients; $68.1 \%)$ and nivolumab (1229/2390 patients; 51.4\%), respectively. Factors positively associated with longer overall therapy duration were: oral fluoropyrimidine in first line (hazard ratio [95\% confidence interval]: 0.63 [0.57-0.69]); trastuzumab in any line $(0.73$ [0.68-0.78]); treatment at a designated cancer hospital (0.89 [0.84-0.94]); dietary consultation within 1 month before/ after start of first line (0.92 [0.86-0.98]); and treatment at a surgical department (0.94 [0.890.99]). Negatively associated factors were: 
edema (1.21 [1.07-1.37]); physical therapy (1.21 [1.12-1.31]); nutritional intervention (1.21 [1.14-1.28]) within 1 month before/after start of first line; thrombosis (1.13 [1.04-1.23]); renal disease (1.11 [1.02-1.21]); age (1.07 [1.021.13]); and peritoneal metastasis/ascites (1.06 [1.01-1.13]).

Conclusions: In real-world treatment practice for AGC in Japan, therapy choice after the recommended first-line chemotherapy was consistent with guidelines. Factors associated with overall therapy duration were identified, which may assist in optimizing treatment sequence.

Keywords: Advanced gastric cancer; Big data; Real-world evidence; Treatment sequence

\section{Key Summary Points}

Why carry out this study?

This study used a large-scale administrative database to give an overview of the real-world treatment pattern after initiating guideline-recommended firstline chemotherapy for advanced gastric cancer in Japan

This study aimed to explore the factors associated with therapy duration and transition from first- to second-line therapy

What was learned from the study?

Oral fluoropyrimidine in first-line therapy, trastuzumab in any line, treatment at a designated cancer hospital, treatment at a surgical department, and dietary consultation at start of first-line therapy were associated with longer overall therapy duration

Physical therapy, nutritional intervention within 1 month before or after the start of first line, edema, thrombosis, renal disease, age, and peritoneal metastasis or ascites were negatively associated with overall therapy duration

In patients with hospitalization records, low body mass index and requirement of assistance for activities of daily living were also negatively associated with overall therapy duration

\section{INTRODUCTION}

Gastric cancer (GC) is the sixth most common cancer and the second leading cause of cancer death globally; there were $>1$ million new cases and 782,685 deaths reported in 2018 [1]. Despite the decreasing trend in mortality rate [2], GC remains one of the leading causes of cancer deaths in Japan; an estimated 43,500 patients died from GC in 2020, accounting for approximately $11.5 \%$ of all cancer deaths [3].

For advanced GC (AGC), the treatment guideline published by the Japanese Gastric Cancer Association (JGCA) recommends the combinations of platinum (cisplatin, oxaliplatin) and fluoropyrimidine (capecitabine, fluorouracil, S-1)-with or without trastuzumab (Tmab) depending on the human epidermal growth factor receptor 2 (HER2) status of the tumor-for first-line (1L) therapy, followed by ramucirumab plus paclitaxel (PTX) for secondline (2L) therapy, and nivolumab or irinotecan for third-line (3L) therapy [4]. For patients for whom these recommended regimens are not suitable, treatment with one or more drugs from the six effective drug categories (i.e., fluoropyrimidine, platinum, taxane, irinotecan, ramucirumab, and nivolumab) should be considered [4].

Recently, the value of real-world evidence has been recognized, and research using large real-world data (RWD) is becoming more common. Findings from RWD can potentially complement those from clinical trials and guide the direction for further studies. As of August 2020, among the available databases in Japan, administrative claims databases contain data on the largest number of patients [5]. Because claims are generated for insurance reimbursement purposes, these databases typically lack the information needed to fully understand the clinical status of patients. Nevertheless, such databases enable the generation of evidence on real-world clinical practice [6]. Several studies have used claims databases in Japan for various cancers [7-11]; however, there has not yet been a large-scale claims database study conducted for AGC in Japan.

The objectives of this study were to provide an overview of the real-world treatment 
patterns of guideline-listed antitumor agents among patients with AGC using a large-scale claims database in Japan and to explore the potential factors associated with overall therapy duration and transition to subsequent therapies.

\section{METHODS}

\section{Study Design}

This was a retrospective study using the Medical Data Vision Co., Ltd. (MDV) database (Tokyo, Japan), which is a de-identified database of health insurance claims collected from hospitals in Japan that use the Diagnostic Procedure Combination (DPC) system. DPC was originally developed as a per-day flat-sum payment system for acute inpatient care [12]. As of April 2020, 1757 hospitals have participated in the DPC system [13, 14], including 447 governmentdesignated cancer hospitals [15]. As of November 2019, the MDV database contained data of approximately 29.5 million patients from 393 DPC hospitals that contracted with MDV and approved research use of their data. The MDV database includes both in- and outpatient claims for diagnosis, medications, and medical procedures and information about the treating hospital and the patient's age and sex. It also provides some clinical information from discharge summaries of hospitalized patients such as height, weight, and the 10-item Barthel activities of daily living (ADL) index [16]. This study used the data of all patients with at least one diagnosis of any neoplasm (International Statistical Classification of Disease and Related Health Problems, 10th Revision codes C00-D48) between 1 June 2014 and 30 September 2019 (Fig. 1). The claims codes for diagnosis, medications, and procedures used in this study are listed in Supplemental Table S1. This study was conducted in accordance with the ethical principles of the Declaration of Helsinki and Good Pharmacoepidemiology Practices. Ethical review and informed consent were not required because this study used retrospective, de-identified data.

\section{Study Population}

The study population consisted of patients with a confirmed diagnosis of GC during the selection period (1 June 2014 to 31 July 2019) (Fig. 1). Other inclusion criteria were: aged 20 years at the start of $1 \mathrm{~L}$; received one of the combination regimens with platinum and fluoropyrimidine recommended as $1 \mathrm{~L}$ by the JGCA guideline [4]; and had initiated 1L on or after 1 June 2015. JGCA guideline-recommended 1L combination regimens with platinum and fluoropyrimidine are S-1 plus cisplatin, S-1 plus oxaliplatin (SOX), capecitabine plus cisplatin (XP), capecitabine plus oxaliplatin, and fluorouracil plus cisplatin, all with or without Tmab, and fluorouracil, oxaliplatin, and leucovorin [4]. Start of $1 \mathrm{~L}$ therapy was defined as the date of the first prescription of a platinum drug or S-1 if S-1 was prescribed within 10 days before the first prescription date of a platinum drug.

To avoid contamination of perioperative drug treatment as the 1L therapy for AGC, patients were excluded if they had gastric resection within 2 months before the start of $1 \mathrm{~L}$, between the start of $1 \mathrm{~L}$ and the start of $2 \mathrm{~L}$ (defined in the "Treatment Sequence" section below), or between the start of $1 \mathrm{~L}$ and the end of patient data (if $2 \mathrm{~L}$ did not exist). To eliminate patients with cancers other than GC, patients were excluded from the study if they received any antitumor agents other than those listed in the JGCA guideline on or after the start of 1L (Supplemental Table S2) [4]. Other exclusion criteria were a $1 \mathrm{~L}$ duration $<35$ days, because such patients are likely to have deteriorated health status and are unlikely to continue the therapy, and participation in clinical trials of the study drugs not covered by health insurance claims after the start of $1 \mathrm{~L}$.

\section{Measures}

\section{Hospital and Patient Characteristics}

Hospital information including number of beds, hospital type (i.e., a designated cancer hospital or not), and the department that prescribed the antitumor agent at the start of $1 \mathrm{~L}$, and patient 


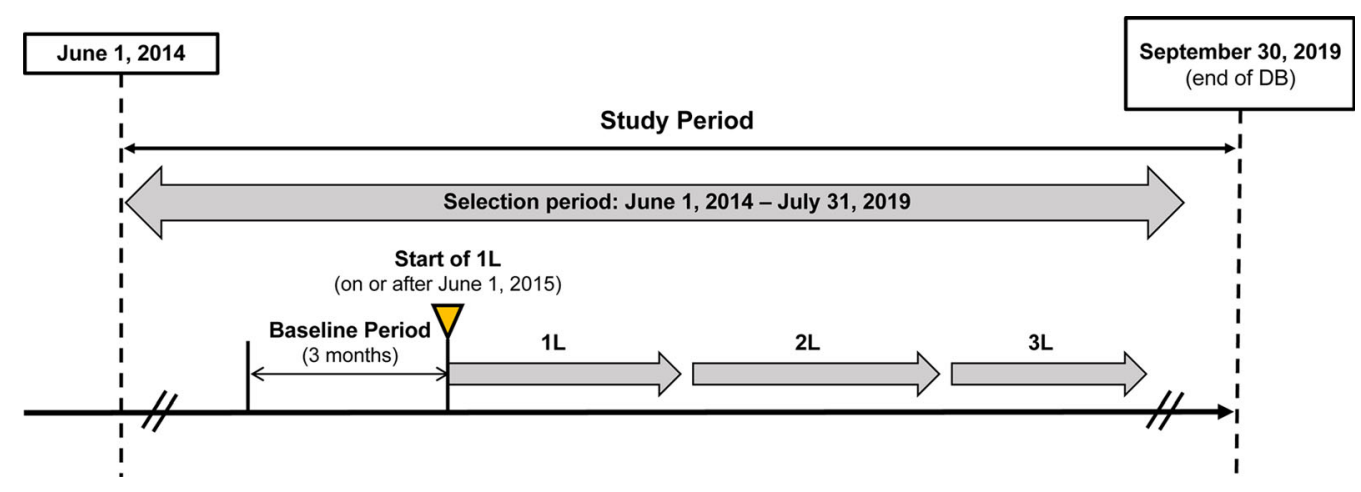

Fig. 1 Study design diagram. $I L$ first line, $2 L$ second line, $3 L$ third line, $D B$ database

characteristics such as age and sex at start of $1 \mathrm{~L}$ and $2 \mathrm{~L}$ were assessed. Other information assessed during the baseline period (defined as 3 months before the start of $1 \mathrm{~L}$, including the start date of $1 \mathrm{~L}$ ) and during $1 \mathrm{~L}$ therapy included history of hospitalization, selected comorbidities (hypertension, diabetes, liver disease, thrombosis, ischemic heart disease, renal disease, neuropathy, edema, hemorrhoids), and metastasis (peritoneal metastasis or ascites, liver metastasis, lymph node metastasis, and lung metastasis). In addition, gastroduodenal stenting between the start of the baseline period and the start of 2L was assessed. Tmab use in any line, which was regarded as a surrogate for HER2 status, was also assessed because histological findings, including HER2 status, are not available in the MDV database. For patients who had a history of hospitalization, weight and height information was collected to calculate body mass index (BMI), and the results of the 10-item Barthel ADL index [16] were collected to measure functional independence. ADL was defined as "independent" if all 10 items were recorded as independent and "dependent" if any items were recorded as not independent. For patients with any ADL items missing, ADL was defined as "missing." Baseline ADL and BMI data were collected from the discharge summary from the last hospitalization that occurred within 3 months prior to the start of $1 \mathrm{~L}$ therapy. $1 \mathrm{~L}$ ADL and BMI data were collected from the discharge summary from the last hospitalization that occurred during 1L therapy. ADL and BMI data were used for the respective analyses for those patients who had both baseline and $1 \mathrm{~L}$ data available.

\section{Treatment Sequence}

The $1 \mathrm{~L}$ regimen was defined as any combination of JGCA guideline-listed antitumor agents (Supplemental Table S2) that included a platinum and a fluoropyrimidine prescribed within 35 days after the start of $1 \mathrm{~L}$, except for Tmab, which was still included in the $1 \mathrm{~L}$ regimen even if it was added after the first 35 days and before the end of $1 \mathrm{~L}$ therapy. A subsequent line of therapy (i.e., 2L, 3L) was defined as any antitumor agent listed in the JGCA guideline that had not been administered in the prior therapy. Each line of therapy was judged to be ended when a different agent not included in the prior regimen was started or when all antitumor agents in the regimen were not administered for $>90$ days. The start of a subsequent line of therapy was defined as the date of the first prescription of any JGCA guideline-listed agents after the end of the previous line.

\section{Therapy Duration}

Durations of each line of therapy were calculated as the time between the start and the last administration date of the JGCA guideline-listed agents included in the regimen of that line. For oral agents, the last administration date was estimated as the last date of prescription plus the number of days' supply minus 1 day. Similarly, overall therapy duration was calculated from the start of $1 \mathrm{~L}$ to the end of the last line in the study period, and therapy duration from the 
start of $2 \mathrm{~L}$ was calculated from the start of $2 \mathrm{~L}$ to the end of the last line in the study period. Patients were considered to be continuing the line and were censored at the last administration date of the line if there were $<90$ days between the end of the line and the end of individual patient data without a subsequent line of therapy. The proportion of patients who received subsequent therapy was evaluated using the number of patients who discontinued a line (i.e., patients who were not censored).

\section{Healthcare Interventions and Laboratory Tests} Healthcare interventions and laboratory tests performed within 1 month before or after the start of $1 \mathrm{~L}$ therapy were analyzed. These included: tumor marker tests (alpha fetoprotein, cancer antigen 19-9, carcinoembryonic antigen); guidance and management for cancer patients (counseling and consultations provided in writing by experienced healthcare professionals); dietary consultation (instruction about eating); nutritional intervention (prescription or infusion of nutritional drugs); and physical therapy (planning and execution of rehabilitations).

\section{Statistical Analysis}

Hospital and patient characteristics were presented using descriptive statistics, with categorical variables presented as $n$ (\%) and continuous variables presented as mean and standard deviation. Median (95\% confidence interval $[\mathrm{CI}]$ ) overall therapy durations and durations of $1 \mathrm{~L}, 2 \mathrm{~L}$, and from the start of $2 \mathrm{~L}$ to last administration of all JGCA guideline-listed antitumor agents were estimated using the Kaplan-Meier method. Cox regression analysis was conducted to identify factors associated with overall therapy duration in the whole cohort and was used to evaluate the impact of ADL and BMI in patients who had valid ADL and BMI data available during the baseline period or during $1 \mathrm{~L}$. Multiple logistic regression analysis was also performed to identify factors associated with transitioning to a $2 \mathrm{~L}$ regimen, in both the whole cohort and patients with valid $\mathrm{ADL}$ and BMI data, and regimen choice in
2L in patients who discontinued $1 \mathrm{~L}$ in the whole cohort. Subgroup analyses for therapy duration from the start of $2 \mathrm{~L}$ to the end of the last line in the study period were conducted in patients who received ramucirumab plus taxane (either PTX or nab-PTX) versus other regimens in $2 \mathrm{~L}$ therapy using patient demographic and clinical characteristics (age [70 years], sex, metastasis, comorbidities or different therapies, duration of $1 \mathrm{~L}$ therapy [ $<150$ days, 150 days], and prior gastroduodenal stenting). Furthermore, regimens used as 3L therapy before and after the approval of nivolumab for AGC in Japan in September 2017 [17] were compared. Sample selection and creation of analytic variables were performed using the Instant Health Data platform (Panalgo, Boston, MA, USA). Statistical analyses were undertaken with $\mathrm{R}$, version 3.2.1 ( $\mathrm{R}$ Foundation for Statistical Computing, Vienna, Austria). Imputation of missing data was not performed.

\section{RESULTS}

\section{Patient Selection}

Of the $10,562,749$ patients with at least one diagnosis of a neoplasm in the full database obtained from MDV (April 2008 to September 2019), 333,910 patients had a claims record of confirmed diagnosis of GC during the selection period. Of these, 17,099 patients were aged 20 years and started the JGCA guideline-recommended platinum- and fluoropyrimidinebased 1L regimens in June 2015 or later. After excluding the patients who met the exclusion criteria, 10,581 patients were identified for this study (Fig. 2).

\section{Hospital and Patient Characteristics}

Most patients were treated at a designated cancer hospital $(8410 / 10,581 ; 79.5 \%)$ and at an internal medicine $(5462 / 10,581 ; 51.6 \%)$ or surgery department $(5035 / 10,581 ; \quad 47.6 \%)$ (Table 1). Prior surgery was received by $32.9 \%$ $(1655 / 5035)$ of patients treated at a surgery department and 7.7\% (423/5462) of those treated at an internal medicine department. Mean age was 67.9 years, with $73.9 \%(7814 / 10,581)$ of 


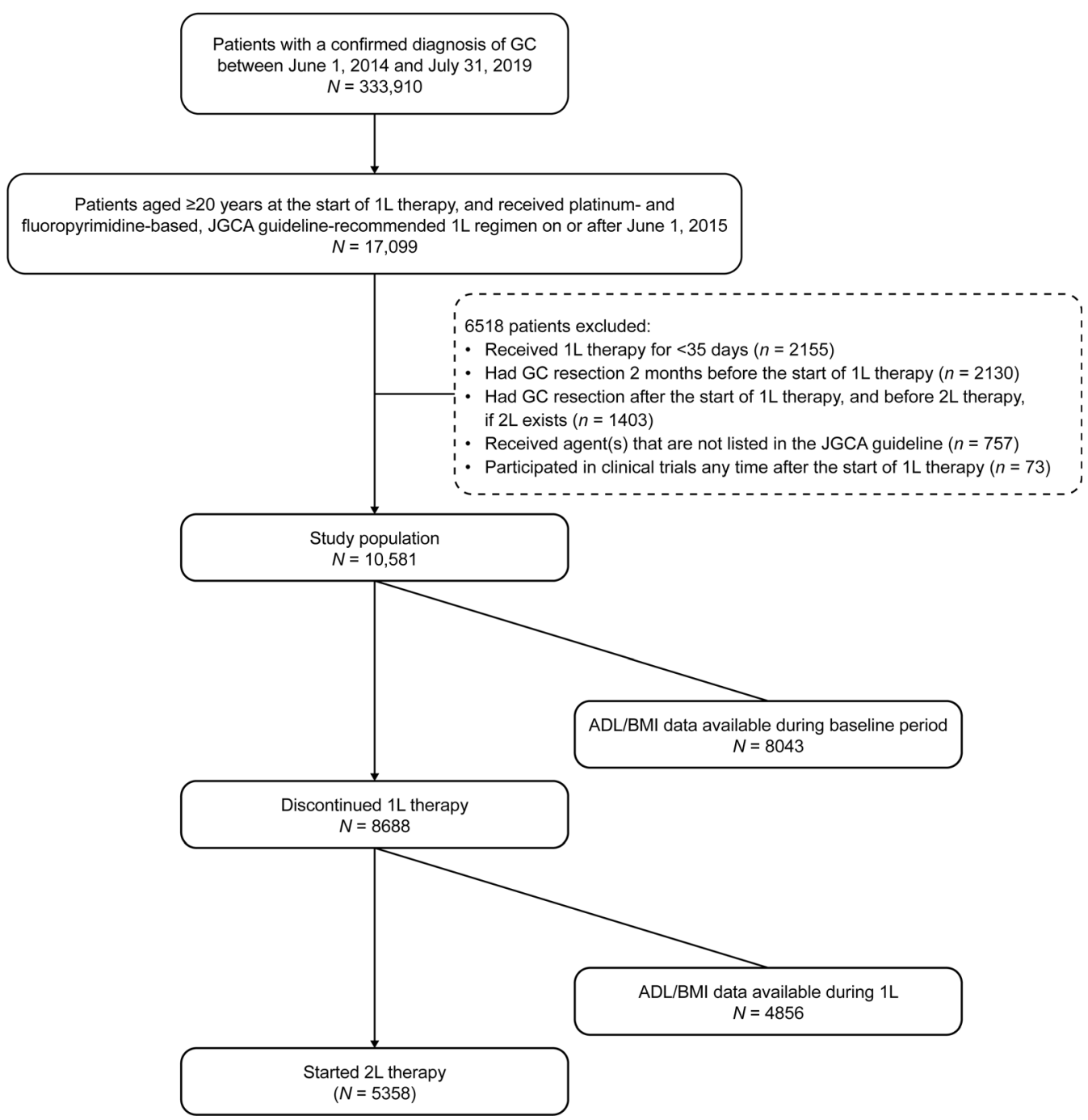

Fig. 2 Flow chart of the study cohort. Note: the $N$ s for ADL/BMI data available during baseline period and during $1 \mathrm{~L}$ represent the number of patients who had both

patients being male. Among patients who had ADL $(N=8088)$ and BMI $(N=8248)$ data available during the baseline period, $91.7 \%$ (7416/8088) of patients had an ADL index of "independent" (i.e., all 10 ADL items were recorded as "independent"), and the mean BMI was $21.2 \mathrm{~kg} / \mathrm{m}^{2}$. The proportion of patients who received Tmab in any line was $14.7 \%(1557 / 10,581)$. Most characteristics were similar between patients with and without Tmab; however, the proportions of patients who were male (with Tmab: 80.5\%; without Tmab: $72.7 \%$ ) and those with liver metastasis (with Tmab: 33.5\%; without
ADL and BMI data available. $I L$ first line, $2 L$ second line, $A D L$ activity of daily living, $B M I$ body mass index, $G C$ gastric cancer, JGCA Japanese Gastric Cancer Association

Tmab: $17.4 \%$ ) were numerically higher in patients with Tmab, and the proportion with peritoneal metastasis or ascites was numerically higher in patients without Tmab (with Tmab: 14.9\%; without Tmab: 24.3\%; Supplemental Table S3).

\section{Treatment Pattern from $1 L$ to $3 L$ Therapy}

There were 10,581 patients who received $1 \mathrm{~L}$, 5358 patients who received $2 \mathrm{~L}$, and 2390 patients who received 3L. Overall, the most common $1 \mathrm{~L}, 2 \mathrm{~L}$, and $3 \mathrm{~L}$ regimens were SOX (4327/10,581; 40.9\%), ramucirumab plus PTX 
Table 1 Hospital and patient characteristics of the study population $(N=10,581)$

\begin{tabular}{|c|c|}
\hline Hospital/patient characteristics ${ }^{a}$ & \\
\hline Number of beds in hospital & \\
\hline$<200$ beds & $451(4.3)$ \\
\hline $200-499$ beds & $5568(52.6)$ \\
\hline$\geq 500$ beds & $4562(43.1)$ \\
\hline Designated cancer hospital & $8410(79.5)$ \\
\hline Medical department & \\
\hline Internal medicine & $5462(51.6)$ \\
\hline Surgery & $5035(47.6)$ \\
\hline Others or unknown & $84(0.8)$ \\
\hline Age, years, mean $(\mathrm{SD})$ & $67.9(9.7)$ \\
\hline Age $\geq 70$ years & $5081(48.0)$ \\
\hline Sex & \\
\hline Male & $7814(73.9)$ \\
\hline Female & $2767(26.2)$ \\
\hline Trastuzumab use in any line & $1557(14.7)$ \\
\hline Metastasis sites ${ }^{\mathrm{b}}$ & \\
\hline Peritoneal metastasis or ascites & $2421(22.9)$ \\
\hline Liver metastasis & $2087(19.7)$ \\
\hline Lymph node metastasis & $1831(17.3)$ \\
\hline Lung metastasis & $478(4.5)$ \\
\hline Comorbidities $^{\mathrm{b}}$ & \\
\hline Hypertension & $3256(30.8)$ \\
\hline Diabetes & $2501(23.6)$ \\
\hline Liver disease & $1845(17.4)$ \\
\hline Thrombosis & $955(9.0)$ \\
\hline Ischemic heart disease & $860(8.1)$ \\
\hline Renal disease & $846(8.0)$ \\
\hline Neuropathy & $769(7.3)$ \\
\hline Edema & $359(3.4)$ \\
\hline Hemorrhoids & $320(3.0)$ \\
\hline Body mass index ${ }^{\mathrm{b}}$ & \\
\hline Data available & $8248(78.0)$ \\
\hline
\end{tabular}

Table 1 continued

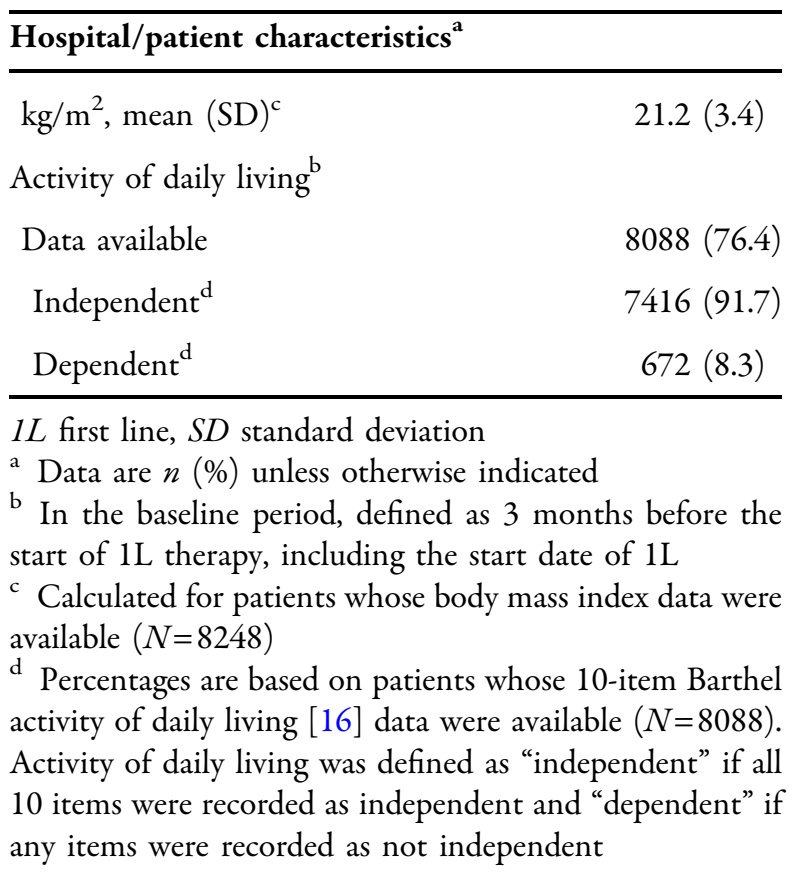

(2990/5358; 55.8\%), and nivolumab (1229/ 2390; 51.4\%), respectively (Fig. 3). During 1L therapy, $6701 \quad(63.3 \%)$ and $3880 \quad(36.7 \%)$ patients received $1 \mathrm{~L}$ regimens that included oxaliplatin (SOX: 40.9\%; XELOX: 12.0\%; FOLFOX: 4.4\%; SOX, Tmab: 4.1\%; XELOX, Tmab: 2.0\%; FOLFOX, Tmab: $<0.1 \%)$ and cisplatin (SP: 23.8\%; XP, Tmab: 5.8\%; FP: 3.3\%; SP, Tmab: 2.3\%; XP: 1.4\%; FP, Tmab: 0.1\%), respectively. Among patients who received $1 \mathrm{~L}$ regimens without Tmab $(N=9069)$ and with Tmab $(N=$ $1512)$, the most common $1 \mathrm{~L}$ regimen was SOX $(4327 / 9069 ; 47.7 \%)$ and XP plus Tmab (608/ $1512 ; 40.2 \%)$, respectively. During 2L therapy, $68.1 \%(3650 / 5358)$ of patients received ramucirumab plus a taxane, either PTX (2990/5358; $55.8 \%)$ or nab-PTX $(660 / 5358 ; 12.3 \%)$. Before the approval of nivolumab for AGC, irinotecan was the most common 3L therapy (361/643; $56.1 \%)$, followed by ramucirumab plus PTX (49/ 643; 7.6\%; Supplemental Table S4). After the approval of nivolumab, nivolumab was the most common 3L therapy (1229/1747; 70.4\%), and only $11.1 \%(193 / 1747)$ of patients received irinotecan (Supplemental Table S5). Less than one-third $(3153 / 10,581 ; 29.8 \%)$ of patients 


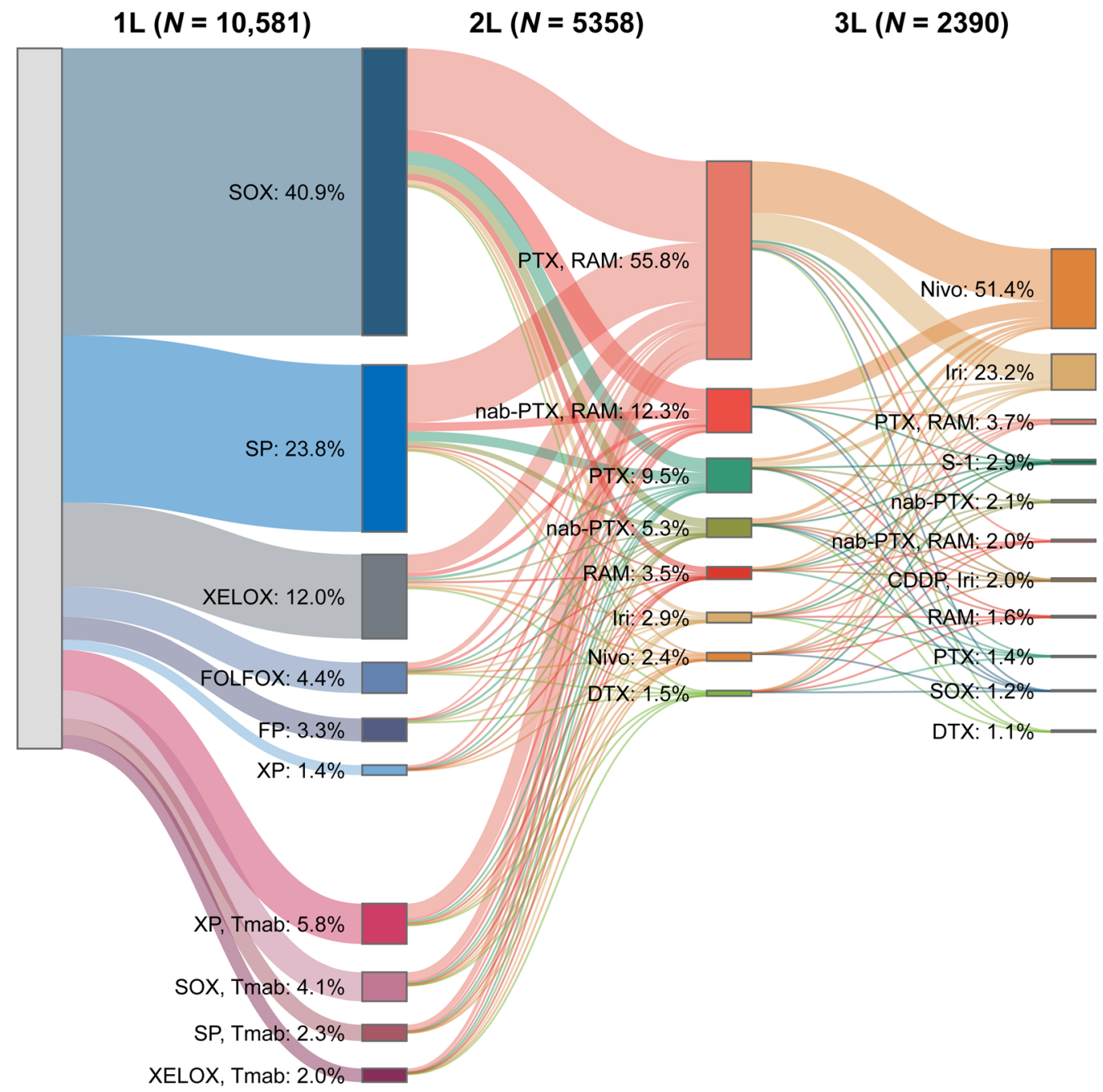

Fig. 3 Treatment sequence of antitumor drug treatment in the study population with or without trastuzumab. This figure is a modification of a previous work by Hironaka et al. [42], presented at the virtual American Society of Clinical Oncology (ASCO) Gastrointestinal Cancer Symposium 2021. Note: the figure does not include very minor sequences because it is not possible to make them visible. $I L$ first line, $2 L$ second line, $3 L$ third line, CDDP

received guidance and management, and fewer received nutritional intervention $(1953 / 10,581$; $18.5 \%)$, dietary consultation $(1762 / 10,581$; $16.7 \%)$, or physical therapy $(1065 / 10,581$; $10.1 \%$ ) within 1 month before or after the start of 1L therapy (Supplemental Table S6). cisplatin, DTX docetaxel, FOLFOX fluorouracil and oxaliplatin and leucovorin, $F P$ fluorouracil and cisplatin, Iri irinotecan, nab-PTX nanoparticle-albumin-bound paclitaxel, Nivo nivolumab, $P T X$ paclitaxel, $R A M$ ramucirumab, SOX S-1 and oxaliplatin, SP S-1 and cisplatin, Tmab trastuzumab, XELOX capecitabine and oxaliplatin, $X P$ capecitabine and cisplatin

\section{Duration of 1L Therapy and Overall Therapy Duration}

In the study population $(N=10,581)$, median duration of $1 \mathrm{~L}$ therapy was 5.8 months and median overall therapy duration was 10.2 months (Supplemental Table S7). Compared with patients without Tmab in any line, those with Tmab had numerically longer duration of 1L therapy (median: 5.5 months vs. 
6.9 months, respectively) and overall therapy duration (median: 9.8 months vs. 13.3 months, respectively; Supplemental Table S7). Cox regression analysis revealed that positive factors associated with overall therapy duration were: oral fluoropyrimidine use in $1 \mathrm{~L}$ (hazard ratio [95\% CI]: 0.63 [0.57-0.69]); Tmab use in any line $(0.73$ [0.68-0.78]); treatment at a designated cancer hospital (0.89 [0.84-0.94]); dietary consultation within 1 month before or after the start of $1 \mathrm{~L}(0.92$ [0.86-0.98]); and treatment at a surgery department (0.94 [0.89-0.99]) (Table 2). Negative factors were: edema (1.21 [1.07-1.37]); physical therapy $(1.21[1.12-1.31])$, or nutritional intervention (1.21 [1.14-1.28]) within 1 month before or after the start of $1 \mathrm{~L}$; thrombosis (1.13 [1.04-1.23]); renal disease (1.11 [1.02-1.21]); age 70 years (1.07 [1.02-1.13]); and peritoneal metastasis or ascites (1.06 [1.011.13]) (Table 2). In the subset analysis limited to patients with ADL and BMI data available in the baseline period $(N=8043)$, dependent ADL and a BMI $18.5 \mathrm{~kg} / \mathrm{m}^{2}$ at baseline were identified as additional negative factors (Supplemental Table S8).

\section{Transition to $2 L$ After Discontinuing $1 L$ \\ Therapy}

Of 8688 patients who discontinued 1L, 5358 $(61.7 \%)$ received 2L therapy, with a higher proportion in patients with Tmab in any line than in those without Tmab $(68.2 \%$ vs. $60.6 \%$; Supplemental Table S7). Multiple logistic regression analysis revealed that positive factors associated with the transition to $2 \mathrm{~L}$ therapy were: oral fluoropyrimidine use in 1L (odds ratio [95\% CI]: 1.90 [1.58-2.27]); duration of $1 \mathrm{~L}$ 150 days (1.50 [1.37-1.64]); peritoneal metastasis or ascites (1.31 [1.18-1.46]); Tmab use in any line (1.24 [1.09-1.42]); liver metastasis (1.23 [1.10-1.38]); treatment at a designated cancer hospital (1.21 [1.09-1.36]); dietary consultation (1.14 [1.01-1.29]); and lymph node metastasis (1.12 [1.00-1.26]) (Table 3). Conversely, negative factors included: physical therapy $(0.71$ [0.61-0.82]); edema (0.75 [0.64-0.87]); age 70 years $(0.78[0.71-0.85])$; and treatment at a surgery department (0.82 [0.74-0.89]) (Table 3). In the subset analysis limited to patients with ADL and BMI data available in 1L $(N=4856)$, dependent ADL and BMI $18.5 \mathrm{~kg} / \mathrm{m}^{2}$ in $1 \mathrm{~L}$ were identified as additional negative factors (Supplemental Table S9).

\section{Use of Ramucirumab Plus Taxane in $2 L$ Therapy}

As mentioned above, ramucirumab plus taxane was the most common regimen in patients who started 2L therapy (Fig. 3). Multiple logistic regression analysis revealed that among patients who started 2L therapy $(N=5358)$, positive factors significantly associated with the use of ramucirumab plus taxane in 2L were: oral fluoropyrimidine in $1 \mathrm{~L}$ (odds ratio [95\% CI]: 3.04 [2.33-3.96]); duration of 1L 150 days (1.23 [1.09-1.38]); and treatment at a designated cancer hospital (1.24 [1.07-1.44]) (Supplemental Table S10). Conversely, negative factors were prior gastroduodenal stenting (0.44 [0.31-0.63]) and pre-existing comorbidities, including thrombosis (0.74 [0.62-0.89]), hemorrhoids (0.75 [0.58-0.97]), and ischemic heart disease (0.75 [0.60-0.93]) (Supplemental Table S10).

Duration of $2 L$ and Therapy Duration from $2 L$ In patients who started 2L therapy $(N=5358)$, median duration of $2 \mathrm{~L}$ therapy was 3.0 months (Table 4). Median therapy duration from the start of $2 \mathrm{~L}$ to the end of the last line in the study period was 5.6 months, and the proportion of patients who received $3 \mathrm{~L}$ after discontinuing $2 \mathrm{~L}$ therapy was $52.9 \% \quad(2390 / 4522$; Table 4). Patients who received ramucirumab plus taxane in $2 \mathrm{~L}$ compared with patients who received other $2 \mathrm{~L}$ regimens had longer duration of $2 \mathrm{~L}$ therapy (median: 3.6 vs. 2.0 months), longer therapy duration from 2L (median: 6.7 vs. 3.5 months), and received 3L more frequently (57.5\% vs. $43.6 \%$; Table 4$)$. However, no statistical comparison was performed because of a potential imbalance in patient backgrounds. In all analyzed subgroups, therapy duration from $2 \mathrm{~L}$ was numerically longer in patients who received ramucirumab plus taxane in $2 \mathrm{~L}$ than in patients receiving other regimens (Supplemental Table S11). 
Table 2 Cox regression analysis of factors associated with overall therapy duration ${ }^{a}$ (study population; $N=10,581$ )

\begin{tabular}{|c|c|c|c|c|}
\hline Covariate & & $\mathbf{H R}^{\mathrm{b}}$ & $95 \% \mathrm{CI}$ & $p$ value \\
\hline Designated cancer hospital & Yes vs. no & 0.89 & $0.84-0.94$ & $<0.001$ \\
\hline \multirow[t]{2}{*}{ Medical department } & Others/unknown vs. internal medicine & 1.15 & $0.91-1.47$ & 0.238 \\
\hline & Surgery vs. internal medicine & 0.94 & $0.89-0.99$ & 0.011 \\
\hline Age & $\geq 70$ vs. $<70$ & 1.07 & $1.02-1.13$ & 0.003 \\
\hline Sex & Male vs. female & 1.00 & $0.95-1.06$ & 0.907 \\
\hline Trastuzumab use in any line & Yes vs. no & 0.73 & $0.68-0.78$ & $<0.001$ \\
\hline \multicolumn{5}{|l|}{ Metastasis sites $^{\mathrm{c}}$} \\
\hline Peritoneal metastasis or ascites & Yes vs. no & 1.06 & $1.01-1.13$ & 0.031 \\
\hline Liver metastasis & Yes vs. no & 1.05 & $0.99-1.12$ & 0.091 \\
\hline Lymph node metastasis & Yes vs. no & 0.97 & $0.91-1.03$ & 0.383 \\
\hline Lung metastasis & Yes vs. no & 1.09 & $0.97-1.22$ & 0.142 \\
\hline \multicolumn{5}{|l|}{ Comorbidities $^{c}$} \\
\hline Hypertension & Yes vs. no & 1.02 & $0.96-1.07$ & 0.550 \\
\hline Diabetes & Yes vs. no & 0.98 & $0.93-1.04$ & 0.525 \\
\hline Liver disease & Yes vs. no & 0.96 & $0.90-1.02$ & 0.205 \\
\hline Thrombosis & Yes vs. no & 1.13 & $1.04-1.23$ & 0.003 \\
\hline Ischemic heart disease & Yes vs. no & 1.00 & $0.91-1.09$ & 0.969 \\
\hline Renal disease & Yes vs. no & 1.11 & $1.02-1.21$ & 0.021 \\
\hline Neuropathy & Yes vs. no & 0.98 & $0.89-1.07$ & 0.660 \\
\hline Edema & Yes vs. no & 1.21 & $1.07-1.37$ & 0.003 \\
\hline Hemorrhoids & Yes vs. no & 1.11 & $0.98-1.27$ & 0.110 \\
\hline \multicolumn{5}{|l|}{$1 \mathrm{~L}$ therapy } \\
\hline Oxaliplatin & Yes vs. no & 0.98 & $0.93-1.03$ & 0.480 \\
\hline Oral fluoropyrimidine & Yes vs. no & 0.63 & $0.57-0.69$ & $<0.001$ \\
\hline
\end{tabular}

Received healthcare interventions and laboratory tests within 1 month before or after start of $1 \mathrm{~L}$ therapy

\begin{tabular}{lcrrr} 
AFP & Yes vs. no & 1.07 & $0.97-1.18$ & 0.203 \\
CA19-9 & Yes vs. no & 1.02 & $0.84-1.23$ & 0.846 \\
CEA & Yes vs. no & 0.95 & $0.79-1.15$ & 0.599 \\
Guidance and management for cancer patients & Yes vs. no & 0.98 & $0.93-1.03$ & 0.470 \\
Dietary consultation & Yes vs. no & 0.92 & $0.86-0.98$ & 0.009 \\
Nutritional intervention & Yes vs. no & 1.21 & $1.14-1.28$ & $<0.001$ \\
\hline
\end{tabular}


Table 2 continued

\begin{tabular}{|c|c|c|c|c|}
\hline Covariate & & $\mathbf{H R}^{\mathbf{b}}$ & $95 \% \mathrm{CI}$ & $p$ value \\
\hline Physical therapy & Yes vs. no & 1.21 & $1.12-1.31$ & $<0.001$ \\
\hline \multirow{3}{*}{\multicolumn{5}{|c|}{$\begin{array}{l}\text { IL first line, } A F P \text { alpha-fetoprotein, } C A 19-9 \text { cancer antigen } 19-9, C E A \text { carcinoembryonic antigen, } C I \text { confidence interval, } \\
H R \text { hazard ratio, } J G C A \text { Japanese Gastric Cancer Association } \\
\text { a Overall therapy duration was defined as the time between the start of } 1 \mathrm{~L} \text { therapy and the date of the last administration } \\
\text { date of all JGCA guideline-listed agents }\end{array}$}} \\
\hline & & & & \\
\hline & & & & \\
\hline \multicolumn{5}{|c|}{$\begin{array}{l}\text { Covariates with } \mathrm{HR}<1.0 \text { were positive factors associated with overall therapy duration; those with an } H R>1.0 \text { were } \\
\text { negative factors }\end{array}$} \\
\hline
\end{tabular}

\section{DISCUSSION}

Medical databases allow access to a large amount of RWD retrospectively, reducing the burden of data collection compared with clinical trials [6]. Furthermore, clinical trials are conducted under controlled conditions with eligibility criteria, limiting the generalizability of their findings. This was the first real-world treatment pattern analysis in patients with AGC using a large-scale database in Japan. Therapy choices after the recommended $1 \mathrm{~L}$ chemotherapy were generally in line with the Japanese guideline [4]. Tmab use in any line (surrogate for HER2 status), oral fluoropyrimidine in 1L therapy, treatment at a designated cancer hospital, and dietary consultation at the start of $1 \mathrm{~L}$ therapy were associated with longer overall therapy duration and a higher proportion of patients receiving 2L therapy. In contrast, presence of edema, receiving physical therapy, and age 70 years were identified as factors that are negatively associated with both overall therapy duration and transition to $2 \mathrm{~L}$ therapy. These factors could be taken into consideration when planning the treatment strategy for a patient in clinical practice.

In this study, we evaluated therapy duration and transition to subsequent therapy as the main treatment outcomes. Endpoints commonly used in clinical trials for oncology, such as tumor response and progression-free survival (PFS), are difficult to evaluate in retrospective studies, especially with administrative databases [18]. In the MDV database, death events only during hospitalization are captured, making evaluation of overall survival (OS) also challenging. However, associations between treatment duration and survival endpoints such as PFS and OS have shown moderate to good correlation [19-21]. For example, in patients with advanced hepatocellular carcinoma in Japan, significant correlation between total duration of administration of all tyrosine kinase inhibitors and OS was observed [22]. For AGC patients in Japan, the reported survival time after discontinuing therapies is relatively short compared with the overall therapy duration $[23,24]$, suggesting an association between overall therapy duration and OS, although further verification is required. Transition to subsequent therapy has been reported to be associated with better survival [25-27], and a meta-analysis concluded that AGC patients treated with 3L therapy had significantly longer OS compared with patients treated with best supportive care only [28]. The JGCA guideline also recommends using all effective drug classes as much as possible [4], further supporting the benefit of treating AGC with multiple therapy lines, which can be represented by the transition to subsequent therapy and used as one of the real-world endpoints in our study.

In this study, factors associated with longer overall therapy duration included the use of Tmab, which was considered a surrogate marker for HER2 status. This was consistent with the results from clinical trials that reported the survival benefits of Tmab in HER2-positive AGC [29], showing better prognosis in patients with HER2-positive AGC compared with those with HER2-negative AGC [30]. Oral fluoropyrimidine use in $1 \mathrm{~L}$ therapy was also associated with 
Table 3 Multiple logistic regression analysis of factors associated with transition from $1 \mathrm{~L}$ to $2 \mathrm{~L}$ therapy (patients who discontinued 1L: $N=8688$ )

\begin{tabular}{|c|c|c|c|c|}
\hline Covariate & & $\mathrm{OR}^{\mathrm{a}}$ & $95 \% \mathrm{CI}$ & $p$ value \\
\hline Designated cancer hospital & Yes vs. no & 1.21 & $1.09-1.36$ & $<0.001$ \\
\hline \multirow[t]{2}{*}{ Medical department } & Others/unknown vs. internal medicine & 0.79 & $0.50-1.26$ & 0.323 \\
\hline & Surgery department vs. internal medicine & 0.82 & $0.74-0.89$ & $<0.001$ \\
\hline Age & $\geq 70$ vs. $<70$ & 0.78 & $0.71-0.85$ & $<0.001$ \\
\hline Sex & Male vs. female & 0.93 & $0.84-1.03$ & 0.166 \\
\hline Trastuzumab use in any line & Yes vs. no & 1.24 & $1.09-1.42$ & 0.002 \\
\hline \multicolumn{5}{|l|}{ Metastasis sites ${ }^{\mathrm{b}}$} \\
\hline Peritoneal metastasis or ascites & Yes vs. no & 1.31 & $1.18-1.46$ & $<0.001$ \\
\hline Liver metastasis & Yes vs. no & 1.23 & $1.10-1.38$ & $<0.001$ \\
\hline Lymph node metastasis & Yes vs. no & 1.12 & $1.00-1.26$ & 0.046 \\
\hline Lung metastasis & Yes vs. no & 1.03 & $0.84-1.27$ & 0.775 \\
\hline \multicolumn{5}{|l|}{ Comorbidities ${ }^{\mathrm{b}}$} \\
\hline Hypertension & Yes vs. no & 0.94 & $0.85-1.03$ & 0.189 \\
\hline Diabetes & Yes vs. no & 1.01 & $0.90-1.12$ & 0.910 \\
\hline Liver disease & Yes vs. no & 0.97 & $0.87-1.08$ & 0.547 \\
\hline Thrombosis & Yes vs. no & 0.88 & $0.77-1.02$ & 0.081 \\
\hline Ischemic heart disease & Yes vs. no & 0.90 & $0.77-1.06$ & 0.222 \\
\hline Renal disease & Yes vs. no & 0.99 & $0.86-1.14$ & 0.879 \\
\hline Neuropathy & Yes vs. no & 0.93 & $0.82-1.06$ & 0.299 \\
\hline Edema & Yes vs. no & 0.75 & $0.64-0.87$ & $<0.001$ \\
\hline Hemorrhoids & Yes vs. no & 1.01 & $0.83-1.24$ & 0.908 \\
\hline \multicolumn{5}{|l|}{$1 \mathrm{~L}$ therapy } \\
\hline Oxaliplatin & Yes vs. no & 0.96 & $0.87-1.06$ & 0.444 \\
\hline Oral fluoropyrimidine & Yes vs. no & 1.90 & $1.58-2.27$ & $<0.001$ \\
\hline Duration of $1 \mathrm{~L}$ therapy & $\geq 150$ days vs. $<150$ days & 1.50 & $1.37-1.64$ & $<0.001$ \\
\hline \multicolumn{5}{|c|}{ Received healthcare interventions and laboratory tests within 1 month before or after the start of $1 \mathrm{~L}$ chemotherapy } \\
\hline AFP & Yes vs. no & 1.18 & $0.98-1.43$ & 0.087 \\
\hline CA19-9 & Yes vs. no & 0.90 & $0.63-1.27$ & 0.544 \\
\hline CEA & Yes vs. no & 1.17 & $0.83-1.65$ & 0.373 \\
\hline Guidance and management for cancer patients & Yes vs. no & 1.05 & $0.95-1.16$ & 0.327 \\
\hline Dietary consultation & Yes vs. no & 1.14 & $1.01-1.29$ & 0.038 \\
\hline Nutritional intervention & Yes vs. no & 0.90 & $0.81-1.01$ & 0.086 \\
\hline
\end{tabular}


Table 3 continued

\begin{tabular}{|c|c|c|c|}
\hline Covariate & $\mathrm{OR}^{\mathrm{a}}$ & $95 \%$ CI & $p$ value \\
\hline Physical therapy & 0.71 & $0.61-0.82$ & $<0.001$ \\
\hline
\end{tabular}

Table 4 Duration of therapy and proportion of patients who received $3 \mathrm{~L}$ therapy after discontinuing $2 \mathrm{~L}$ therapy in the patients who started $2 \mathrm{~L}$ therapy

\begin{tabular}{|c|c|c|c|}
\hline & $\begin{array}{l}\text { Ramucirumab plus taxane } \\
\text { in } 2 L\end{array}$ & $\begin{array}{l}\text { Other regimens in } \\
2 L\end{array}$ & Overall \\
\hline Started 2L therapy, $n$ & 3650 & 1708 & 5358 \\
\hline $\begin{array}{l}\text { Duration of } 2 \mathrm{~L} \text { therapy, months }{ }^{\mathrm{b}} \text {, median }(95 \% \\
\text { CI) }\end{array}$ & $3.6(3.5-3.8)$ & $2.0(1.9-2.3)$ & $3.0(3.0-3.3)$ \\
\hline $\begin{array}{l}\text { Therapy duration from } 2 \mathrm{~L}^{\mathrm{c}} \text {, months }{ }^{\mathrm{b}} \text {, median } \\
(95 \% \mathrm{CI})\end{array}$ & $6.7(6.3-7.0)$ & $3.5(3.3-4.0)$ & $5.6(5.4-5.9)$ \\
\hline \multicolumn{4}{|l|}{ Transitioned from $2 \mathrm{~L}$ to $3 \mathrm{~L}$ therapy } \\
\hline Received 3L therapy, $n / n^{\mathrm{d}}(\%)$ & $1728 / 3004(57.5)$ & $662 / 1518(43.6)$ & $\begin{array}{l}2390 / 4522 \\
(52.9)\end{array}$ \\
\hline
\end{tabular}

$2 L$ second line, $3 L$ third line, $C I$ confidence interval, JGCA Japanese Gastric Cancer Association

${ }^{a}$ Either paclitaxel or nanoparticle-albumin-bound paclitaxel

${ }^{\text {b }}$ Calculated as 28 days $=1$ month

c Therapy duration from $2 \mathrm{~L}$ was defined as the time from the start of $2 \mathrm{~L}$ to the last estimated administration date of all JGCA guideline-listed agents

${ }^{d}$ Patients who discontinued $2 \mathrm{~L}$ and had data available $\geq 90$ days after the end of each treatment line or transitioned to the next line were included

longer overall therapy duration, which might be because patients who can take oral medicines generally have relatively better health status. Treatment in a surgical department was another positive factor, although the reason for this association is unclear, and unmeasured confounding factors may be involved because, conversely, it is a negative factor for the transition to $2 \mathrm{~L}$ therapy. One notable difference between the departments was that the proportion of prior surgery was substantially higher in patients treated at surgical departments compared with those treated at internal medical departments (32.9\% vs. $7.7 \%$, respectively). This might indicate that more patients at surgery departments had recurrent disease after surgery, which may be associated with better prognosis than unresectable advanced disease because gastrectomy has been reported to be a favorable prognostic factor [31]. Poor nutritional status is reported to increase the risk of drug toxicity and may lead to treatment discontinuation and decreased survival [27, 32]. A prospective study also reported that AGC 
patients with low nutritional risk had longer median survival than patients with high nutritional risk (31.9 vs. 25.7 months, $p<0.001$ ), and improvement of nutritional status may prolong survival [33]. In this study, dietary consultation was a positive factor for longer therapy duration; however, physical therapy and nutritional intervention were negative factors. Although the extent of supportive care may vary considerably between hospitals, dietary consultation was likely to be provided when patients still had better health status, whereas nutritional intervention and physical therapy are more intensive interventions indicative of poor health status and therefore would have been provided only when patients' health status had deteriorated. Similarly, other factors indicative of poor health status (older age, metastases and comorbidities, low BMI, and dependent ADL) were also associated with a shorter overall therapy duration. Furthermore, factors associated with the transition to subsequent therapies were similar to those associated with overall therapy duration; patients with factors indicative of poor health status were less likely to transition to the subsequent therapy. Collectively, these findings highlight the potential importance of preserving and maintaining good health status by providing proactive patient support early to extend therapy duration and transitioning and ultimately to improve survival.

In this study, $68.1 \%$ of patients received ramucirumab plus taxane in 2L therapy. Prior gastroduodenal stenting was a strong negative factor for using ramucirumab in $2 \mathrm{~L}$, which is reasonable considering the potential associations between stenting and gastrointestinal perforation in patients treated with ramucirumab [34]. Pre-existing ischemic heart disease and thrombosis were also factors against ramucirumab use in $2 \mathrm{~L}$, possibly because various cardiovascular events are known class effects associated with antiangiogenic agents $[35,36]$. However, hypertension was not a negative factor, even though hypertension is a known adverse event associated with ramucirumab [37-39]. This may be because ramucirumab-related hypertension is usually well managed using standard antihypertensive medications [39]. In this study, patients treated with ramucirumab plus taxane showed numerically longer therapy duration from $2 \mathrm{~L}$ compared with other regimens; similar trends were observed in all patient subgroups that were analyzed. However, as the MDV database lacks important clinical information to adjust for patient background factors, a formal statistical comparison between treatments could not be conducted in this study.

There were some limitations in this study. Although the MDV database is one of the largest databases in Japan, it covers only a portion of DPC hospitals, which may have caused selection bias. Only the patients who received the guideline-recommended $1 \mathrm{~L}$ regimens were included in the study, and some patients were excluded for use of drugs in later lines of therapy that are not listed in the guidelines. As the database is unable to track patients beyond each hospital, patients might have been lost or counted multiple times if they changed hospitals, and therefore the lines of therapy may not be accurately reflected. Diagnostic information was based only on claims, and treatment lines were defined using a prespecified analysis algorithm; therefore, this information may not be entirely accurate. The database is poorly populated with clinical and disease-related information [40], including prognostic factors (e.g., performance status, number of metastatic organs, histology, recurrent vs. de novo advanced disease) and cancer stages, which may lead to confounding by indication when evaluating the effectiveness of different drugs [41]. Finally, ADL and BMI data were only available for patients who were hospitalized during the evaluated time periods.

\section{CONCLUSIONS}

The use of a large-scale database in this study allowed us to describe the real-world practice with guideline-listed antitumor agents for the treatment of AGC in Japan. The choice of treatment regimens after the recommended $1 \mathrm{~L}$ chemotherapies consistently followed the current Japanese guideline. Factors associated with overall therapy duration and transition to 
subsequent therapy were identified, which may guide further research to explore optimal sequential treatment and supportive care for AGC.

\section{ACKNOWLEDGEMENTS}

Funding. Eli Lilly Japan K.K. (Kobe, Japan), manufacturer/licensee of ramucirumab, sponsored this study and was involved in the study design, data acquisition, data analysis, and preparation of the manuscript and funded the Rapid Service and Open Access fees.

Medical Writing, Editorial and Other Assistance. Medical writing assistance was provided by Prudence Stanford, PhD, and Hana Nomura, BPharm (Hons), of ProScribe-Envision Pharma Group, and was funded by Eli Lilly Japan K.K. ProScribe's services complied with international guidelines for Good Publication Practice (GPP3).

Authorship. All named authors meet the International Committee of Medical Journal Editors (ICMJE) criteria for authorship for this article, take responsibility for the integrity of the work as a whole, and have given their approval for this version to be published.

Author Contributions. All authors were involved in the study design and participated in the interpretation of study results and in the drafting, critical revision, and approval of the final version of the manuscript. Yoshinori Tanizawa and Zhihong Cai conducted the statistical analysis.

Prior Presentation. This study was presented at the virtual American Society of Clinical Oncology (ASCO) Gastrointestinal Cancer Symposium 2021, held on 15-17 January 2021.

Disclosures. Yoshinori Tanizawa, Zhihong Cai, and Yongzhe Piao are employees and/or minor shareholders of Eli Lilly Japan K.K. Yoshito Komatsu has received honoraria and/or research grants from Eli Lilly Japan K.K., Taiho
Pharmaceutical Co., Ltd., Chugai Pharmaceutical Co., Ltd., Takeda Pharmaceutical Co., Ltd., Bayer Yakuhin, Ltd., Bristol-Myers Squibb Co., Sanofi/Aventis, Merck, Yakult Honsha Co., Ltd., Ono Pharmaceutical Co., Ltd., Nipro Corp., Moroo Co., Ltd., Asahi Kasei Corp., Mitsubishi Tanabe Pharma Corp., Otsuka Pharmaceutical Co., Ltd., Medical Review Co., Ltd., Daiichi Sankyo Co., Ltd., Merck Sharp \& Dohme, NanoCarrier Co., Ltd., Eisai Co., Ltd., Shionogi \& Co., Ltd., Iqvia Services Japan K.K., Parexel International Corp., Astellas Pharma Inc., Mediscience Planning Inc., Sumitomo Dainippon Pharma Co. Ltd., A2 Healthcare Corp., Incyte, Eli Lilly and Co., and BeiGene Ltd. Shuichi Hironaka has received personal fees, honoraria, and/or research grants from BristolMyers Squibb K.K., Ono Pharmaceutical Co., Ltd., Taiho Pharmaceutical Co., Ltd., Yakult Honsha Co., Ltd., Daiichi Sankyo Co., Eli Lilly and Co., Chugai Pharmaceutical Co., Ltd., Nippon Kayaku Co., Ltd., Tsumura \& Co., Sanofi, Merck, AstraZeneca PLC, MSD K.K., Pfizer Inc., Boehringer Ingelheim International $\mathrm{GmbH}$, Toyama Chemical Co., Kyowa Kirin Co., Ltd., Chugai Pharmaceutical Co., Ltd., Astellas Pharma Inc., Yakult Honsha Co., Ltd., Shionogi \& Co., Ltd., and Takeda Pharmaceutical Co., Ltd. Narikazu Boku has received honoraria and/or research grants from Ono Pharmaceutical Co., Ltd., Takeda Pharmaceutical Co. Ltd., Taiho Pharmaceutical Co., Ltd., Bristol-Myers Squibb K.K., and Bristol-Myers Squibb Co.

Compliance with Ethics Guidelines. This study was conducted in accordance with the ethical principles of the Declaration of Helsinki and Good Pharmacoepidemiology Practices. Retrospective, de-identified data were used in this study, and therefore ethical review and informed consent were not required. This article is based on previously conducted studies and does not contain any new studies with human participants or animals performed by any of the authors.

Data Availability. The datasets generated and/or analyzed during the current study are not publicly available because the data in this 
study were commercially obtained from Medical Data Vision Co., Ltd., and were used under licence. Data can be made available from Medical Data Vision Co., Ltd., upon request.

Open Access. This article is licensed under a Creative Commons Attribution-NonCommercial 4.0 International License, which permits any non-commercial use, sharing, adaptation, distribution and reproduction in any medium or format, as long as you give appropriate credit to the original author(s) and the source, provide a link to the Creative Commons licence, and indicate if changes were made. The images or other third party material in this article are included in the article's Creative Commons licence, unless indicated otherwise in a credit line to the material. If material is not included in the article's Creative Commons licence and your intended use is not permitted by statutory regulation or exceeds the permitted use, you will need to obtain permission directly from the copyright holder. To view a copy of this licence, visit http:// creativecommons.org/licenses/by-nc/4.0/.

\section{REFERENCES}

1. Bray F, Ferlay J, Soerjomataram I, Siegel RL, Torre LA, Jemal A. Global cancer statistics 2018: GLOBOCAN estimates of incidence and mortality worldwide for 36 cancers in 185 countries. CA Cancer J Clin. 2018;68(6):394-424.

2. Carioli G, Malvezzi M, Bertuccio P, et al. Cancer mortality in the elderly in 11 countries worldwide, 1970-2015. Ann Oncol. 2019;30(8):1344-55.

3. Center for Cancer Control and Information Services. National Cancer Center. Projected Cancer Statistics, 2018. https://ganjoho.jp/en/public/ statistics/short_pred.html. Accessed Aug 24, 2020.

4. Japanese Gastric Cancer Association. Japanese gastric cancer treatment guidelines 2018 (5th edition). Gastric Cancer. 2021;24(1):1-21.

5. Pharmacoepidemiology \& Database Taskforce, Japanese Society for Pharmacoepidemiology. Survey of Japanese databases in Japan available for clinical/pharmacoepidemiology. http://www.jspe. jp/committee/020/0210/. Accessed Sep 18, 2020.
6. Sherman RE, Anderson SA, Dal Pan GJ, et al. Realworld evidence-What is it and what can it tell us? N Engl J Med. 2016;375(23):2293-7.

7. Cheung S, Hamuro Y, Mahlich J, Nakayama M, Tsubota A. Treatment pathways of Japanese prostate cancer patients-a retrospective transition analysis with administrative data. PLoS ONE. 2018;13(4):e0195789.

8. Wang F, Mishina S, Takai S, et al. Systemic treatment patterns with advanced or recurrent nonsmall cell lung cancer in Japan: a retrospective hospital administrative database study. Clin Ther. 2017;39(6):1146-60.

9. Goto Y, Yamamoto N, Masters ET, et al. Treatment sequencing in patients with anaplastic lymphoma kinase-positive non-small cell lung cancer in Japan: a real-world observational study. Adv Ther. 2020;37 (7):3311-23.

10. Mori A, Hashimoto K, Koroki Y, Wu DB, Masumori $\mathrm{N}$. The correlation between metastasis-free survival and overall survival in non-metastatic castration resistant prostate cancer patients from the Medical Data Vision claims database in Japan. Curr Med Res Opin. 2019;35(10):1745-50.

11. Tsutsué S, Tobinai K, Yi J, Crawford B. Nationwide claims database analysis of treatment patterns, costs and survival of Japanese patients with diffuse large B-cell lymphoma. PLoS One. 2020;15(8):e0237509.

12. Matsuda S, Fujimori K, Fushimi K. Development of casemix based evaluation system in Japan. Asian Pac J Dis Manag. 2010;4(3):55-66.

13. Hayashida K, Murakami G, Matsuda S, Fushimi K. History and profile of Diagnosis Procedure Combination (DPC): development of real data collection system for acute inpatient care in Japan. J Epidemiol. 2021;31(1):1-11.

14. Ministry of Health, Labour and Welfare. 令和 2 年度 診療報酬改定の概要 (DPC/PDPS). https://www. mhlw.go.jp/content/12400000/000603946.pdf. Accessed Nov 17, 2020.

15. Foundation for Promotion of Cancer Research. Cancer statistics in Japan-2021. https://ganjoho.jp/ data/reg_stat/statistics/brochure/2021/cancer_ statistics_2021.pdf. Accessed Jun 15, 2021.

16. Wade DT, Collin C. The Barthel ADL Index: a standard measure of physical disability? Int Disabil Stud. 1988;10(2):64-7.

17. Pharmaceuticals and Medical Devices Agency. 医薬 品インタビューフォーム: OPDIVO ${ }^{\circledR}$ I.V. Infusion 20mg100mg200mg240mg. https://www.info.pm 
da.go.jp/go/interview/1/180188_4291427A1024_1_ 030_1F.pdf. Accessed Aug 20, 2021.

18. Griffith SD, Tucker M, Bowser B, et al. Generating real-world tumor burden endpoints from electronic health record data: comparison of RECIST, radiology-anchored, and clinician-anchored approaches for abstracting real-world progression in non-small cell lung cancer. Adv Ther. 2019;36(8):2122-36.

19. Stewart M, Norden AD, Dreyer N, et al. An exploratory analysis of real-world end points for assessing outcomes among immunotherapy-treated patients with advanced non-small-cell lung cancer. JCO Clin Cancer Inform. 2019;3:1-15.

20. Yang G, Ma D, Xu H, et al. Treatment duration as a surrogate endpoint to evaluate the efficacy of crizotinib in sequential therapy for patients with advanced ALK-positive non-small cell lung cancer: a retrospective, real-world study. Cancer Med. 2019;8(13):5823-30.

21. Blumenthal GM, Gong Y, Kehl K, et al. Analysis of time-to-treatment discontinuation of targeted therapy, immunotherapy, and chemotherapy in clinical trials of patients with non-small-cell lung cancer. Ann Oncol. 2019;30(5):830-8.

22. Hiraoka A, Kumada T, Atsukawa M, et al. Important clinical factors in sequential therapy including lenvatinib against unresectable hepatocellular carcinoma. Oncology. 2019;97(5):277-85.

23. Kimura M, Kawachi S, Go M, et al. Effect of the timing of discontinuation of last-line chemotherapy on patient prognosis in advanced and recurrent gastric cancer. Mol Clin Oncol. 2019;10(1):173-9.

24. Otsuka M, Koyama A, Matsuoka H, et al. Early palliative intervention for patients with advanced cancer. Jpn J Clin Oncol. 2013;43(8):788-94.

25. Takashima A, Iizumi S, Boku N. Survival after failure of first-line chemotherapy in advanced gastric cancer patients: differences between Japan and the rest of the world. Jpn J Clin Oncol. 2017;47(7):5839.

26. Iizumi S, Takashima A, Sakamaki K, Morita S, Boku N. Survival impact of post-progression chemotherapy in advanced gastric cancer: systematic review and meta-analysis. Cancer Chemother Pharmacol. 2018;81(6):981-9.

27. Hamamoto Y, Piao Y, Makiyama A. Achieving sequential therapy in advanced gastric cancer: the importance of appropriate patient management for the elderly and/or those with ascites. Gastric Cancer. 2020;23(3):363-72.
28. Zheng Y, Zhu XQ, Ren XG. Third-line chemotherapy in advanced gastric cancer: a systematic review and meta-analysis. Medicine (Baltimore). 2017;96 (24):e6884.

29. Bang Y-J, Van Cutsem E, Feyereislova A, et al. Trastuzumab in combination with chemotherapy versus chemotherapy alone for treatment of HER2positive advanced gastric or gastro-oesophageal junction cancer (ToGA): a phase 3, open-label, randomised controlled trial. Lancet. 2010;376 (9742):687-97.

30. Shitara K, Yatabe Y, Matsuo K, et al. Prognosis of patients with advanced gastric cancer by HER2 status and trastuzumab treatment. Gastric Cancer. 2013;16(2):261-7.

31. Wang J, Qu J, Li Z, et al. A prognostic model in metastatic or recurrent gastric cancer patients with good performance status who received first-line chemotherapy. Transl Oncol. 2016;9(3):256-61.

32. Salati M, Di Emidio K, Tarantino V, Cascinu S. Second-line treatments: moving towards an opportunity to improve survival in advanced gastric cancer? ESMO Open. 2017;2(3):e000206.

33. Qiu M, Zhou YX, Jin Y, et al. Nutrition support can bring survival benefit to high nutrition risk gastric cancer patients who received chemotherapy. Support Care Cancer. 2015;23(7):1933-9.

34. Jung M, Ryu MH, Oh DY, et al. Efficacy and tolerability of ramucirumab monotherapy or in combination with paclitaxel in gastric cancer patients from the Expanded Access Program Cohort by the Korean Cancer Study Group (KCSG). Gastric Cancer. 2018;21(5):819-30.

35. Touyz RM, Lang NN, Herrmann J, van den Meiracker AH, Danser AHJ. Recent advances in hypertension and cardiovascular toxicities with vascular endothelial growth factor inhibition. Hypertension. 2017;70(2):220-6.

36. Abdel-Qadir H, Ethier J-L, Lee DS, Thavendiranathan P, Amir E. Cardiovascular toxicity of angiogenesis inhibitors in treatment of malignancy: a systematic review and meta-analysis. Cancer Treat Rev. 2017;53:120-7.

37. Fuchs CS, Tomasek J, Yong CJ, et al. Ramucirumab monotherapy for previously treated advanced gastric or gastro-oesophageal junction adenocarcinoma (REGARD): an international, randomised, multicentre, placebo-controlled, phase 3 trial. Lancet. 2014;383(9911):31-9.

38. Wilke H, Muro K, Van Cutsem E, et al. Ramucirumab plus paclitaxel versus placebo plus paclitaxel in patients with previously treated advanced 
gastric or gastro-oesophageal junction adenocarcinoma (RAINBOW): a double-blind, randomised phase 3 trial. Lancet Oncol. 2014;15(11):1224-35.

39. Arnold D, Fuchs CS, Tabernero J, et al. Meta-analysis of individual patient safety data from six randomized, placebo-controlled trials with the antiangiogenic VEGFR2-binding monoclonal antibody ramucirumab. Ann Oncol. 2017;28(12):293242 .

40. Brookhart MA, Stürmer T, Glynn RJ, Rassen J, Schneeweiss $\mathrm{S}$. Confounding control in healthcare database research: challenges and potential approaches. Med Care. 2010;48(6 Suppl):S114-20.

41. McMahon AD, MacDonald TM. Design issues for drug epidemiology. Br J Clin Pharmacol. 2000;50 (5):419-25.

42. Hironaka S, Komatsu Y, Tanizawa Y, Cai Z, Piao Y, Boku N. Factors associated with treatment duration from start of second-line ramucirumab plus paclitaxel or nab-PTX for advanced gastric cancer: realworld evidence from Japanese Claim Database. J Clin Oncol. 2021;39(3_suppl):177. 